\title{
Erratum to: Taxonomy of rare genetic metabolic bone disorders
}

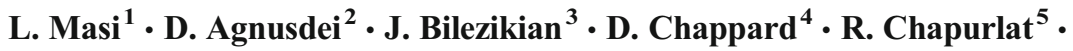 \\ L. Cianferotti ${ }^{1}$ - J.-P. Devolgelaer ${ }^{6}$ - A. El Maghraoui ${ }^{7}$ - S. Ferrari ${ }^{8}$ - K. Javaid $^{9}$. \\ J.-M. Kaufman ${ }^{10}$ • U. A. Liberman ${ }^{11}$ - G. Lyritis ${ }^{12}$ - P. Miller ${ }^{13}$ • N. Napoli ${ }^{14}$ • \\ E. Roldan ${ }^{15}$ - S. Papapoulos ${ }^{16}$ - N. B. Watts $^{17}$ - M. L. Brandi ${ }^{1}$
}

Published online: 14 September 12015

(C) International Osteoporosis Foundation and National Osteoporosis Foundation 2015

\section{Erratum to: Osteoporos Int \\ DOI 10.1007/s00198-015-3188-9}

Correction of publisher's error: In the original publication, Fig. 1 appeared in the place of Fig. 8 and vice versa. The article has now been updated to correct this error.

The online version of the updated original article can be found at $\mathrm{http}: / / \mathrm{dx}$. doi.org/10.1007/s00198-015-3188-9.

M. L. Brandi

marialuisa.brandi@unifi.it

1 Metabolic Bone Diseases Unit, Department of Surgery and Translational Medicine, University Hospital of Florence, University of Florence, Florence, Italy

2 Eli Lilly and Co., Florence, Italy

3 College of Physicians and Surgeons, Columbia University, New York, NY, USA

4 GEROM Groupe Etudes Remodelage Osseux et bioMatériaux-LHEA, IRIS-IBS Institut de Biologie en Santé, LUNAM Université, Angers, France

5 INSERM UMR 1033, Department of Rheumatology, Université de Lyon, Hospices Civils de Lyon, Lyon, France

6 Departement de Medicine Interne, Cliniques Universitaires UCL de Saint Luc, Brussels, Belgium

7 Service de Rhumatologie, Hôpital Militaire Mohammed V, Rabbat, Morocco

8 Division of Bone Diseases, Faculty of Medicine, Geneva University Hospital, Geneva, Switzerland

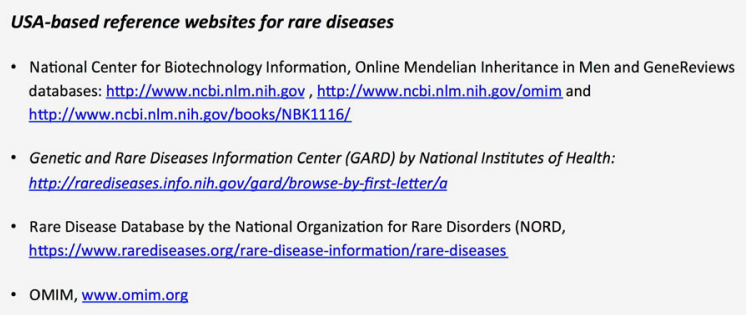

Fig. 1 Main English websites on rare diseases with constantly updated databases (as most recently accessed in January 2015)

9 Oxford NIHR Musculoskeletal Biomedical Research Unit, University of Oxford, Oxford, UK

10 Department of Endocrinology, Ghent University Hospital, Gent, Belgium

11 Department of Physiology and Pharmacology and the Felsenstein Medical Research Center, Sackler School of Medicine, Tel Aviv University, Tel Aviv, Israel

12 Laboratory for the Research of Musculoskeletal System, University of Athens, Athens, Greece

13 Colorado Center for Bone Research, University of Colorado Health Sciences Center, Lakewood, CO, USA

14 Division of Endocrinology and Diabetes, Università Campus Bio-Medico di Roma, Rome, Italy

15 Department of Clinical Pharmacology, Gador SA, Buenos Aires, Argentina

16 Center for Bone Quality, Leiden University Medical Center, Leiden, The Netherlands

17 Mercy Health Osteoporosis and Bone Health Services, Cincinnati, OH, USA 


\section{Biochemical Markers}

- Serum Calcium

- Urinary Calcium

- Serum Phosphate

- Urinary Phosphate

- Serum Magnesium

- Urinary Magnesium

- Parathyroid hormone (PTH)

- Fibroblast growth factor 23 (FGF23)

- 25 hydroxyvitamin $\mathrm{D}_{3}\left[25(\mathrm{OH}) \mathrm{D}_{3}\right]$

- 1,25 dihydroxyvitamin $\mathrm{D}_{3}\left[1-25(\mathrm{OH})_{2} \mathrm{D}_{3}\right]$

- Bone Formation Markers: Total Alkaline Phosphatase, Bone Alkaline Phosphatase, Osteocalcin, Procollagen $1 \mathrm{~N}$-terminal Propeptide (P1NP), Procollagen $1 \mathrm{C}$-termina Propeptide (P1CP)

- Bone Resorption Markers:

Hydroxyproline, Pyridinoline and Deoxypyridinoline, Cross-linked $\mathrm{N}$-telopeptide of type I collagen (NTX), Cross-linked C-telopeptide of type I collagen (CTX)

- Bone Mineralization Markers: Pyridoxal-5'-phosphate

Urinary 4-pyridoxic acid

- Bone Microenvironment Products: Sclerostin, RANK-ligand, Osteprotegerin

\section{Instrumental exams}

- DEXA: Lumbar Spine, hip, wrist, total body

- Ultrasound (US): heel, finger

- Peripheral Quantitative Computed Tomography (pQCT): leg, wrist

- Quantitative Computed Tomography (QCT): spine

- X-rays

- Radiographic vertebral morphometry

- DEXA: Vertebral Fracture Assessment (VFA)

- Bone scintigraphy

- Positron Emission Tomography (PET)

- Magnetic Resonance Imaging (MRI)

\section{Bone Biopsy}

- Histology

- Histomorphometry

In vitro assays
Fig. 8 Biochemical/instrumental exams and in vitro tests for characterizing metabolic bone diseases. Measurements of biochemical indexes and hormones regulating mineral homeostasis can help in confirming or excluding systemic bone metabolic disorders. The assessment of bone turnover is important in order to plan further therapeutic approaches. Bone quantity and quality appraisal and prevalent vertebral fracture assessment may help to refine the metabolic framing of the disease, manifesting with an otherwise evident bone phenotype, and is crucial in the follow-up of the treated patient. Bone biopsy is critical in selected cases for the identification and for differential diagnosis. In vitro assays can be useful to identify supposed functional abnormalities of bone cells and/or matrix proteins (e.g., in collagen-related disorders) 